\title{
Possible mechanism of increasing resistance of the myocardium during combination of post infarction remodeling and diabetes mellitus
}

\author{
Margarita V. Egorova*, Sergey A. Afanasiev, Dina S. Kondratyeva, Boris N. Kozlov, Sergey V. Popov
}

Laboratory of Molecular Cell Pathology and Genetic Diagnosis, Research Institute of Cardiology, Siberian Branch of Russian Academy of Medical Science, Tomsk, Russia; *Corresponding Author: mwegorova@yandex.ru

Received 2 February 2011; revised 21 February 2011; accepted 13 March 2011.

\begin{abstract}
It was shown that the energy metabolism of the heart mitochondria of experimental animals and patients is more resistant to damage at combined postinfarction cardiosclerosis and diabetes in comparison with the individual pathology. We found that the changes of free fatty acid content and conjugation of the processes of oxidation and phosphorylation in heart mitochondria are components of the metabolic stability of myocardium at the combined development of postinfarction cardiosclerosis and diabetes mellitus. Our data demonstrate a direct link between the violations of the processes of oxidative phosphorylation and accumulation of free fatty acids owing to change in activity of endogenous phospholipases, in particularly, mitochondrial phospholipase $A_{2}$. Similar results were obtained for intraoperative biopsy specimens of patients' hearts, and of adult Wistar rats' hearts. We hypothesized that the preservation of energy metabolism is a manifestation of summing up of compensatory processes at development of nonspecific response of cells to damage at the early stages of pathological process.
\end{abstract}

Keywords: Postinfarction Remodeling; Diabetes; Heart Mitochondria; Fatty Acids; Phospholipase $\mathrm{A}_{2}$

\section{INTRODUCTION}

It is well known that many pathologies of the cardiovascular system are accompanied by increased activity of endogenous phospholipases and, as a consequence, by accumulation of free fatty acids [1] which, in turn, provokes uncoupling of the processes of oxidation and phosphorylation in mitochondria [2]. A decrease of insu- lin action on adipose tissue at diabetes mellitus (DM) leads to the increased content of fatty acids in blood and their intake into the myocardial cells. Myocardial consequences of DM even in conditions of adequate oxygen supply of myocardium resemble metabolic imbalance in myocardium of patients with severe coronary heart disease (CHD) [3,4]. At DM, as at CHD, the corresponding complex of pathophysiological changes appears either because of the inability of mitochondria to oxidize the entire volume of incoming fatty acids, or because of critical lowering of coronary blood flow (at CHD), or because of the enhanced transport of fatty acids in the cytosol (at DM) [3,4]. It is logical to suppose that hemodynamic changes on the DM background can increase the probability of lethal outcome that is confirmed by clinical studies $[4,5]$.

However, there is data of manifestation of myocardium resistance to ischemia (in vivo and in vitro) of animals with short term of induced diabetes [6-8]: the induction of diabetes on the background of postinfarction cardiosclerosis preserves, paradoxically, myocardium contractile properties. Animals with combined pathology are characterized by less pronounced changes in glucose levels, body weight and heart $[9,10]$.

The purpose of this study was evaluation of influence of diabetes mellitus and postinfarction remodeling on ability to oxidative phosphorylation of isolated heart mitochondria of animals and humans both in the case of individual pathologies and in the case of their combination.

\section{EXPERIMENTAL PART}

\subsection{Materials}

The work was accomplished on mature male rats of Wistar and intraoperative biopsy samples of patients.

\subsubsection{Animals}

5 groups for 8 rats were formed from animals: 
Group I - control animals,

Groups II and V - animals with induced DM,

Group III - rats after coronary occlusion,

Group IV - rats with combined pathology: DM was induced after coronary occlusion after 2 weeks.

\subsubsection{Patients}

Studies were carried out on intraoperative atrial biopsies of male patients aged 52 - 69 years with underlying diagnosis of coronary heart disease (CHD). The biopsy specimens were divided into two groups:

The first group - CHD: patients with a diagnosis of coronary heart disease (12 samples).

Underlying disease: CHD, exertional angina, functional class III (FC). Multivascular atherosclerosis of coronary arteries. Postinfarction cardiosclerosis (4 - 6 years after acute myocardial infarction). Chronic heart failure (HF) by classifying the New York Heart Association (NYHA) II, with preserved LV systolic function: left ventricular ejection fraction (LVEF) $>45 \%(59 \%-$ $65 \%$ ). Background pathology: arterial hypertension, degree III, Risk 4, dislipidemia.

The second group - CHD + DII: patients with a diagnosis of CHD with a combination of diabetes mellitus type 2 (6 samples).

Underlying disease: CHD, exertional angina FC III. Postinfarction cardiosclerosis (after acute myocardial infarction $-4-6$ years old). HF NYHA II, LVEF $>45 \%$ (59\% - 65\%). Background pathology: arterial hypertension III, Risk, 4, dislipidemia. Concomitant disease: Diabetes mellitus type 2 , moderate, subcompensated $\left(\mathrm{Hb} \mathrm{A}_{1 \mathrm{C}}\right.$ $7.5 \%$; fasting glucose $10-13 \mathrm{mmol} / \mathrm{L}$, in urine $-40-45$ g/day; cholesterol total $5.2-6.5 \mathrm{mmol} / \mathrm{l}$, low density lipoprotein (LDL) cholesterol $>3.0 \mathrm{mmol} / 1$, high density lipoprotein $\mathrm{HDL}<0.9 \mathrm{mmol} / \mathrm{l})$. Body mass index (BMI) of $35-39 \mathrm{~kg} / \mathrm{m}^{2}$, abdominal obesity 2 .

The duration of the underlying disease from the time of registration of 8 - 10 years, concominant disease-at least 5 years.

Standard treatment: antianginal and antihypertensive medicines, hypolipidemic agents (statin or/and fibrate), and, for diabetes - hypoglycemic agent (metformin 1500 $2000 \mathrm{mg} / \mathrm{d})$.

Myocardial tissue samples of patients were frozen in liquid nitrogen, prior to withstanding them at least 1 hour in cold Krebs-Henseleit buffer containing 20\% dimethyl sulfoxide (DMSO) [2]. Before the experiment, biopsies thawed in warm Krebs-Henseleit buffer and then used as freshly isolated tissue [2].

\subsection{Methods}

\subsubsection{Simulation of Diabetes Mellitus}

The development of diabetes was induced by a single injection of streptozotocin (Sigma, USA) in a dose of 60 $\mathrm{mg} / \mathrm{kg}$, intraperitoneally, diluted ex tempera in 0,01 $\mathrm{mol} / \mathrm{L}$ citrate buffer ( $\mathrm{pH} 4.5$ ) [6]. Diabetes mellitus was verified by an increase of glucose concentration in rat blood by 4,5 times and decrease of body weight of $56 \%$ $(\mathrm{p}<0.05)$ in comparison with the animals injected with citrate buffer. Glucose concentration in blood serum was determined with help of enzymatic - colorimetric test ("Biocon Diagnostic", Germany). The animals in Groups II and IV were included into the study in 4 weeks after the induction of diabetes mellitus, the animals in Group $\mathrm{V}-$ in 6 weeks after.

\subsubsection{Modeling of Postinfarction Cardiosclerosis}

Modeling of postinfarction cardiosclerosis in animals was performed under deep ether anesthesia. The thoracic cage of animals was opened and dissected into the two ribs. After pericardiotomy, the coronary occlusion was performed by ligation in the upper third of the left descending coronary artery. Then, after removing air from the thoracic cage cavity, the wound was sutured in layers. After 40 days myocardial infarction was formed in these animals: a morphological control of changes in the structure of myocardial tissue was performed by means of histological study, as previously described [1]. The animals in this group had myocardial hypertrophy (Figure 1) (the heart size of the operated animals exceeded the heart size of control rats, an average of $80 \%$ ), and the necrosis zone was about $12 \%$ of the total mass of the hypertrophied left ventricle (Table 1). For the experiment we used animals in 6 weeks after coronary occlusion. Mitochondria obtained from control animal hearts (sham-operated animals) were used as a control.

\subsubsection{Measurementes}

Heart mitochondria of patients and animals were re-

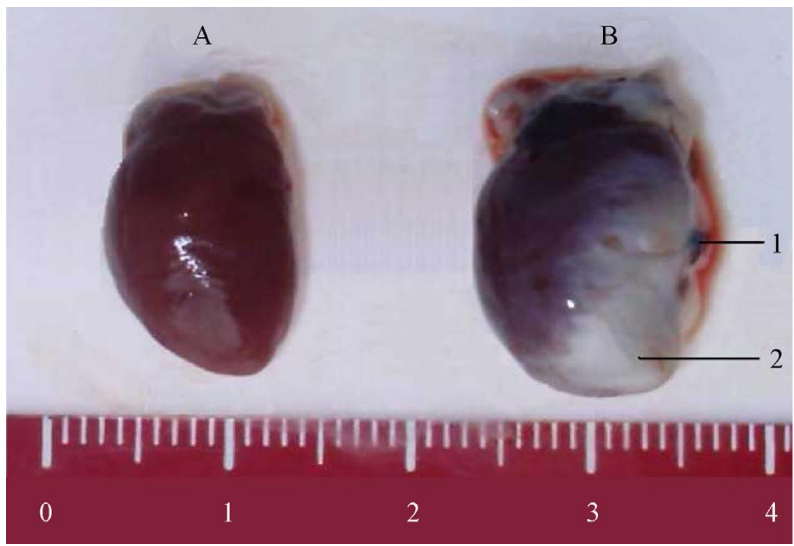

Figure 1. The typical form of rat heart. Note: A - the heart of the control animal, B - the heart of the animal after 40 days after coronary occlusion; 1 - place ligation, 2 - zone scar. 
Table 1. Weights of rats after experimental coronary occlusion.

\begin{tabular}{ccc}
\hline Parameter & $\begin{array}{c}\text { sham-operated } \\
\text { animals }\end{array}$ & $\begin{array}{c}40 \text { days after } \\
\text { coronary occlusion }\end{array}$ \\
\hline Body weight $(\mathrm{g})$ & $287 \pm 23.07$ & $236.6 \pm 3.07$ \\
Heart weight $(\mathrm{mg})$ & $955.9 \pm 44.68$ & $1491.2 \pm 20.96^{*}$ \\
LV weight $(\mathrm{mg})$ & $620.5 \pm 35.37$ & $962.2 \pm 13.09^{*}$ \\
$\begin{array}{c}\text { Weight of the } \\
\text { damaged area }(\mathrm{mg})\end{array}$ & 0 & $109.1 \pm 1.44^{*}$ \\
\hline
\end{tabular}

ceived using standard method of differential centrifugation in sucrose medium containing $(\mathrm{mM}) 300$ of sucrose, 10 EDTA, 8 Tris, pH 7.4 [8]. For storage of mitochondria, we used $250 \mathrm{mM}$ sucrose solution.

The rate of oxygen uptake by mitochondria was determined polarographically by Clark electrode. Measurements were carried out in medium ( $\mathrm{pH} 7.4)$ containing $(\mathrm{mM})$ : sucrose (300), $\mathrm{KCl}(10), \mathrm{KH}_{2} \mathrm{PO}_{4}(5)$, succinate (5), EGTA (1), $\mathrm{MgCl}_{2}(1,2)$, Tris (5). We used the following additives: ADP - $100 \mathrm{uM}$, p-bromophenacyl bromide (BPB) - $15 \mathrm{uM}$, arachidonic acid (AA) - $45 \mu \mathrm{M}$. We used Sigma and ICN reagents.

The respiratory control (RC) was defined as the ratio of the respiration rate at the maximum ATP synthesis to respiration rate in the absence of ATP synthesis [9].

The rate of oxygen consumption is given in $\mathrm{nM} \mathrm{O}_{2}$ per minute per $1 \mathrm{mg}$ of protein. The protein concentration in the sample was determined by the standard Lowry method.

The content of fatty acids was determined in serum, homogenates and mitochondrial suspension by enzymatic endpoint method ("DiaSys Diagnostic Systems", Germany) and calculated on $1 \mathrm{mg}$ of protein.

\subsection{Statistical Methods}

All data are presented as a mean \pm standard error of the mean. The critical level of significance when testing statistical hypotheses $(p)$ was taken 0.05 . In connection with the fact that the distribution law of the studied parameters does not correspond to normal (Shapiro-Wilk test, $p>0.05$ ) law, we used nonparametric criteria to reveal differences in the groups. For independent data (one index in various groups) used rank Mann-Whitney test. For dependent data (various parameters in one group) used Wilcoxon test.

\section{RESULTS AND DISCUSSION}

\subsection{Change of the Content of Fatty Acids in Serum and Myocardium}

It is known that fatty acids are involved in the maintenance of membrane gomeostasis. Reorganization of membrane lipid composition was modulated by "remodeling" of membrane phospholipids with phospholipase A2 and arachidonic acid - this is a quick and subtle regulation of membrane lipid composition in response to changing concentrations and ratios of fatty acids [11]. The change in FFA concentration leads to a change in the permeability of cardiomyocyte membrane, that, in turn, influences functional activity of cardiomyocytes $[12,13]$. We carried out research of fatty acid content in serum, homogenates of the myocardium and in the mitochondria suspension of animals and humans in all groups studied (Table 2). It was found that fatty acid content in blood serum of experimental animals was reliably higher in all experimental groups in relation to control, but in comparison between the experimental groups no reliable differences were observed (Table 2). There was no significant difference between control and experimental animals in the homogenate. The most interesting result was found at comparison of the data of fatty acids content in mitochondrial suspension: a reliable difference in fatty acids content was observed not only in relation of experimental animals to control ones, but also between the groups (Table 2). The comparison of data showed that the smallest difference in fatty acid content in the mitochondrial suspension in relation to control was observed at combination of pathologies.

Similarly, patients with CHD and CHD + DII did not reveal statistically significant differences in fatty acid content in the blood serum and myocardium homogenate, but in a mitochondrial suspension with combination of pathologies much less fatty acid content was observed (Table 2).

\subsection{Mitochondrial Respiration in Studied Groups}

Comparing the initial rate of mitochondrial respiration in the studied groups of animals we found that in all experimental groups, this figure was significantly higher than in the group of control animals (Table 3). In Group II it increased by 4 times, in Group III -more than by 3 times, in Group IV with the combined pathology—only by 2 times. Reducing the $\mathrm{RC}$ value in II-IV groups demonstrated decrease in the conjugation degree of oxidation and phosphorylation at these pathologies, however, in Group IV the uncoupling degree is also less pronounced in comparison with individual pathologies (Table 3).

The initial rate of oxygen uptake by human heart mitochondria at CHD is almost by 2 times higher than at combination CHD + DII (Table 3). Low RC indicates uncoupling of oxidation and phosphorylation in both groups, but lower rate of oxygen consumption (along with higher RC) shows that this uncoupling is less pro- 
Table 2. The content of fatty acids in serum and myocardium of animals and humans.

\begin{tabular}{|c|c|c|c|}
\hline \multirow{2}{*}{ Experimental groups } & \multicolumn{3}{|c|}{ The content of fatty acids (nM per mg protein) } \\
\hline & serum & homogenate & mitochondria \\
\hline \multicolumn{4}{|c|}{ animals } \\
\hline Group I & $0.38 \pm 0.08$ & $1.02 \pm 0.14$ & $0.83 \pm 0.12$ \\
\hline Group II & $1.68 \pm 0.21^{*}$ & $1.51 \pm 0.17$ & $5.83 \pm 1.31^{*} \#^{\wedge}$ \\
\hline Group III & $0.83 \pm 0.14^{*}$ & $1.19 \pm 0.14$ & $2.86 \pm 1.15^{*} \#^{\wedge}$ \\
\hline Group IV & $1.45 \pm 0.35^{*}$ & $1.35 \pm 0.15$ & $1.88 \pm 0.78^{*} \#$ \\
\hline \multicolumn{4}{|c|}{ patients } \\
\hline CHD & $7.35 \pm 0.93$ & $9.33 \pm 1.62$ & $7.2 \pm 1.44 \#$ \\
\hline$C H D+D I I$ & $9.63 \pm 0.81$ & $8.15 \pm 1.34$ & $4.2 \pm 1.36^{\wedge} \#$ \\
\hline
\end{tabular}

Note. The experimental conditions and groups are described in the "Experimental part". * - differences of the results in the column are statistically significant as compared with Group I; \# - differences of the results in each group are statistically significant as compared with each other; $\wedge$ - differences between groups are statistically significant.

Table 3. Rate of oxygen consumption and respiratory control of heart mitochondria of animals and humans.

\begin{tabular}{|c|c|c|c|}
\hline \multirow{2}{*}{ Experimental groups } & \multicolumn{2}{|c|}{ The rate of oxygen consumption, $n M \mathrm{O}_{2}$ per minute per mg of protein. } & \multirow{2}{*}{$R C$} \\
\hline & initially & $+B P B$ & \\
\hline \multicolumn{4}{|c|}{ animals } \\
\hline Group I & $10.5 \pm 1.8$ & $10.6 \pm 1.4$ & $3.4 \pm 0.09$ \\
\hline Group II & $44.7 \pm 2.8^{*}$ & $38.0 \pm 1.7^{*} \#^{\wedge}$ & $2.0 \pm 0.01$ \\
\hline Group III & $35.2 \pm 3.5^{*}$ & $21.1 \pm 2.4^{*} \#^{\wedge}$ & $1.9 \pm 0.02$ \\
\hline Group IV & $20.9 \pm 1.5^{*}$ & $11.6 \pm 1.5 \#^{\wedge}$ & $2.3 \pm 0.05$ \\
\hline \multicolumn{4}{|c|}{ patients } \\
\hline$C H D$ & $33.2 \pm 1.5$ & $18.7 \pm 2.4 \#^{\wedge}$ & $2.0 \pm 0.01$ \\
\hline$C H D+D I I$ & $17.6 \pm 2.1$ & $7.5 \pm 1.2 \#^{\wedge}$ & $2.4 \pm 0.03$ \\
\hline
\end{tabular}

Note. The experimental conditions and groups are described in the "Experimental part". * - differences of the results in the column are statistically significant as compared with Group I; \# - differences of the results in each group are statistically significant when comparing between indexes "initially" and "+ BPB"; ^ - differences between groups are statistically significant.

nounced in the case of a CHD and DII combination (Table 3). Presented data and our earlier observations [10] allow us to assert that at combination of pathologies cardiomyocytes are accompanied by less pronounced violation of the energy metabolism both in animals and in humans.

We have previously suggested and confirmed the assumption that the violation of energy metabolism in rat cardiomyocytes may be related to changes in the accumulation of fatty acids and in the activity of endogenous phospholipases [14]. Inhibition of phospholipase $A_{2}$ by $p$-bromophenacyl bromide (BPB) in postinfarction rat cardiomyocytes resulted in normalization of cellular respiration to the level of normal cardiomyocytes. Activation of phospholipase $A_{2}$ by arachidonic acid or melit- tin in cardiomyocytes of control rats significantly increased the need of cells in oxygen [14].

In this study, in the presence of BPB, we observed a pronounced decrease of the oxygen uptake rate by animals cardiomyocytes mitochondria of the III-IV Groups, while in Group IV this figure was equal to that in control group (Group I) (Table 3). Continuation of this trend is observed for the human heart mitochondria: in the presence of BPB a oxygen uptake rate in patients with CHD and $\mathrm{CHD}+\mathrm{DII}$ decreased by $44 \%$ and $56 \%$ respectively relative to the initial oxygen consumption rate (Table 3 ).

A significant reduction in the oxygen consumption rate by mitochondria of animals and humans with a combination of pathologies in the presence of BPB demonstrates lability of mitochondrial phospholipase $A_{2}$. 
It is the evidence of the greater stability of mitochondrial membrane to damages and, consequently, gives hope that violation of energy metabolism (closely associated with membrane processes) is not irreversible.

\subsection{Mitochondrial Respiration in Groups with Different Terms of Diabetes}

A comparative analysis of oxygen consumption rate by rat heart mitochondria at different stages of streptozotocin-induced diabetes showed that in animals of the Group V a sharp difference of indices is observed not only in comparison with control animals (Group I), but also in comparison with diabetes at an earlier stage (Group II) (Figure 2). Initial oxygen consumption rate in Group $\mathrm{V}$ differs from one of control animals more than by 10 times and more than 3 times greater than this figure in comparison with Group II. The degree of uncoupling of oxidation and phosphorylation becomes dangerously high ( $\mathrm{RC}-1,5)$, indicating a critical violation of energy in cardiomyocytes. Inhibition phospholipase $\mathrm{A}_{2}$ by BPB does not lead to normalization of respiration (Figure 2). When studying the influence of arachidonic acid we observed significant stimulation of oxygen consumption rate of heart mitochondria in Group I and, albeit to a much lesser extent, in Group II (Figure 2). We are prone to consider effect as modulation of phospholipase $\mathrm{A}_{2}$ activity [2]. Lack of stimulating effect of arachidonic acid on oxygen consumption rate in the Group $\mathrm{V}$ (Figure 2), in such a manner, confirms violation of

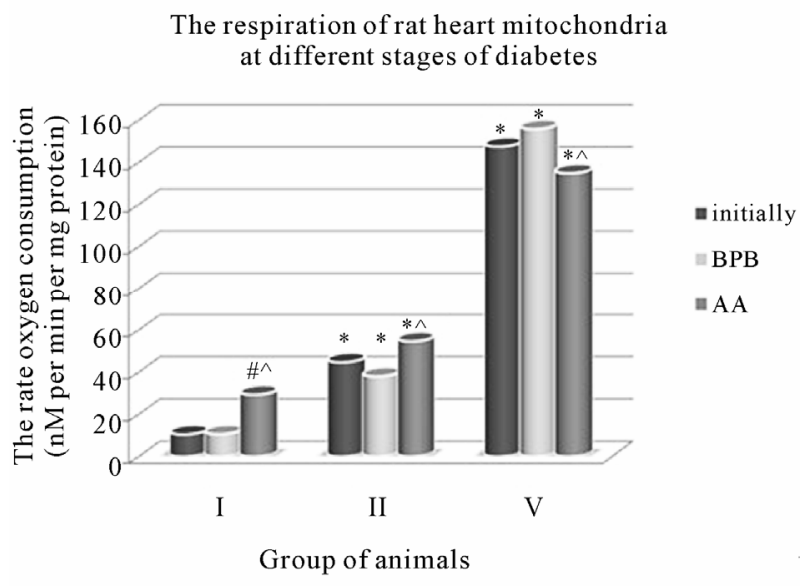

Figure 2. Effect of BPB and arachidonic acid (AA) on the rate of oxygen consumption by mitochondria hearts of rats at different stages of diabetes. Note: The experimental conditions and groups are described in the "Experimental part". * - differences of the results in the column are statistically significant as compared with Group I; \# - differences of the results in each group are statistically significant when comparing between indexes "initially" and "+BPB"; ^ - differences between groups are statistically significant. membrane remodeling and irreversibility of their dam age. In these circumstances, even slight changes in myocardial perfusion inevitably lead to cell death.

Our results and some literature data [6-10], at first glance, are inconsistent with clinical observations about rapidly growing degree of lethal outcome in such combination of pathologies [10,5]. However, it is well known that any disturbing factors action start the process of nonspecific reaction in cells, developing in certain consequence $[15,16]$. Early stages of pathological processes are connected with active start and use of compensatory processes to restore functional activity of a cell, on the later stages this process becomes irreversible $[15,16]$. It is possible that in our case cooperative effect is observed: it is necessary to reveal what specific processes take part in it. Only the fact raises no doubt that these processes are connected with activation of membrane enzymes and reorganization of membrane. It is also possible that along with this, the oxidation processes are switching to alternatives variances, as it occurs, for example, during hypoxia (rapid oxidation of succinic acid) [17].

One of the most powerful natural endogenous mechanisms of adaptation during prolonged ischemia is a "preconditioning phenomenon". A lot of data testifying to multiple levels of organization of this mechanism (see, for example, reviews $[18,19]$ ) has been obtained in last decades. Although our experimental conditions do not meet the requirements of manifestation of the preconditioning phenomenon (alternation of short episodes of sublethal ischaemia and reperfusion), it is possible to draw parallels. At preconditioning, in addition to shortterm adaptive reaction within 1-2 hours, developing delayed, less powerful but more prolonged ( 72 hours) reaction, which was called "second window" [20]. Is it possible that combination of pathologies opens the "third one"'?

\section{CONCLUSIONS}

Thus, the results obtained testify to the fact that disturbance of myocardial energy is the expression of nonspecific reaction of myocardial cells to injury both in case of combined CHD + DII and in case of separate pathologies. Our data demonstrate a direct link between violations of the processes of oxidative phosphorylation and accumulation of free fatty acids due to changes in the activity of endogenous phospholipases, in particular, mitochondrial phospholipase $\mathrm{A}_{2}$.

This article was prepared based on the research funded by the Ministry of Education and Science under the Federal Program "Research and development of prior directions of scientific-technological complex of Russia for 2007-2012" (HA No. 02.527.11.0007) and the grant 
of the 7th Framework Programme of Russia-EU (No. 241558).

\section{REFERENCES}

[1] Kondratieva, D.S., Afanas'ev, S.A., Falaleeva, L.P. and Shakhov, V.P. (2005) Inotropic response of myocardium of rats with postinfarction cardiosclerosis on extrasystolic effect. Bulletin of Experimental Biology and Medicine, 139, 613-616.

[2] Pallotti, F. and Lenaz, G. (2001) Isolation and subfractionation of mitochondria from animal cells and tissue culture lines. Methods Cell Biology, 65, 1-35.

[3] Aleksandrov, A.A. (2003) Diabetic heart: fight for the mitochondria. Consilium Medicum, 5, 509-513.

[4] Kannel, W.B. and McGree, D.L. (1979) Diabetes and cardiovascular disease. The Framingham study. Journal of the American Medical Association, 241, 2035-2038.

[5] Stanley, W.C., Lopaschuk, G.D. and McCormack, J.G. (1997) Regulation of substrate metabolism in diabetic heart. Cardiovascular Research, 34, 25-33.

[6] Nawata, T. Takahashi, N. and Oopie, T. (2002) Cardioprotection by streptozotocin-induced diabetes and insulin against ischemia/reperfusion injury in rats. Journal of Cardiovascular Pharmacology, 40, 491-500.

[7] Chen, H., Shen, W.L. and Wang, X.H. (2006) Paradoxically enhanced heart tolerance to ischemia in type I diabetes and role of increased osmolarity. Clinical and Experimental Pharmacology and Physiology, 33, 910-916.

[8] Dubiley, T.A., Badova, T.A., Migovan, S.A. and Rushkevich, Yu.E. (2007) Influence of ischemia/reperfusion on function of isolated heart in rats of different ages with streptozotocin diabetes. Problems of Ageing and Longevity, 16, 11-21.

[9] Afanas'ev, S.A., Kondratieva, D.S., Tsapko, L.P., Popov, S.V. and Karpov, R.S. (2009) Features inotropic responses of rat myocardium at extrasystolic effect in the combined development of infarction atherosclerosis and diabetes mellitus. Vestnik Arrhythmology, 55, 56-59.

[10] Egorova, M.V., Afanas'ev, S.A., Popov, S.V. and Karpov, R.S. (2010) Adaptive changes at combined development of postinfarction remodeling and diabetes. Bulletin of Experimental Biology and Medicine, 150, 132-135.
[11] Grynberg, A. (1999) The role of lipids in the metabolism of the heart muscle. Medikography, 21, 29-38

[12] Mokhova, E.N. and Haylova, L.S. (2005) Involvement of anion carriers of the inner membrane of mitochondria in the uncoupling action of fatty acids. Biochemistry, Vol. 70, 197-202.

[13] Molchanov, S.N., Lyusov, S.A., Govorin, A.V. and Neverov, I.V. (2005) Serum lipids at various stages and morphofunctional types of heart failure in patients with myocardial infarction. Russian Cardiology Journal, 2, 18-25.

[14] Egorova, M.V., Afanas'ev, S.A. and Popov, S.V. (2008) The role of phospholipase $A_{2}$ in the activation of respiration of isolated cardiomyocytes with infarction cardiosclerosis. Bulletin of Experimental Biology and Medicine, 146, 631-633.

[15] (2010) The sequence of events during the development of non-specific cell responses to injury. In: Novitsky, V.V., Goldberg E.D. and Urazova, O.I., Eds., Pathophysiology, Publishing GEOTAR-Media, Moscow, 1, 59-62.

[16] Lukyanova, L.D. (2004) Mitochondrial dysfunction-a typical pathological process, the molecular mechanism of hypoxia. In: Lukyanova L.D. and Ushakov, I.B., Eds., Problems of Hypoxia: Molecular, Physiological and Medical Aspects, Publi “Origins”, Moscow, 8-18.

[17] Maevsky, E.I., Grishina, E.V., Rosenfeld, A.S., Zyakun, A.M., Kondrashova, M.N. and Vereshchagina, V.M. (2000) Anaerobic formation of succinate and facilitating its oxidation - the possible mechanisms of cell adaptation to hypoxia. Biophysics, 45, 509-513.

[18] Millar, C.G.M., Baxter, G.F. and Thiemermann, C. (1996) Protection of the myocardium by ischaemic preconditioning: mechanisms and therapeutic implications. Pharmacology \& Therapeutics, 69, 143-151.

[19] Yellon, D.M., Dana, A. and Walker, J.M. (1999) Endogenous myocardial protection: the importance of metabolic adaptation (preconditioning). Medikography, 21, 80-84.

[20] Yellon, D.M. and Baxter, G.F. (1995) A "second window of protection" or delayed preconditioning phenomenon: future horizons for myocardial protection? Journal of Molecular and Cellular Cardiology, 27, 1023-1034. 